\title{
Comparison of Human Pilot (Remote) Control Systems in Multirotor Unmanned Aerial Vehicle Navigation
}

\author{
Zainal Rasyid Mahayuddin ${ }^{\# 1}$, Hairina Mohd Jais ${ }^{\# 2}$ and Haslina Arshad ${ }^{\# 3}$

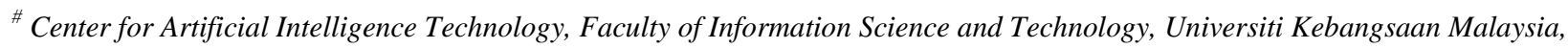 \\ Bangi 43600, Selangor, Malaysia \\ E-mail: ${ }^{1}$ hairinajais@siswa.ukm.edu.my, ${ }^{2}$ zainalr@ukm.edu.my, ${ }^{3}$ haslinarshad@ukm.edu.my
}

\begin{abstract}
This paper concerns about the human pilot or remote control system in UAV navigation. Demands for Unmanned Aerial Vehicle (UAV) are increasing tremendously in aviation industry and research area. UAV is a flying machine that can fly with no pilot onboard and can be controlled by ground-based operators. In this paper, a comparison was made between different proposed remote control systems and devices to navigate multirotor UAV, like hand-controllers, gestures and body postures techniques, and visionbased techniques. The overall reviews discussed in this paper have been studied in various research sources related to UAV and its navigation system. Every method has its pros and cons depends on the situation. At the end of the study, those methods will be analyzed and the best method will be chosen in term of accuracy and efficiency.
\end{abstract}

Keywords - UAV; remote-controller; navigation system; remotely piloted vehicle.

\section{INTRODUCTION}

Nowadays, there has been a rapidly increasing interest in UAV for vary purposes. The UAV, an acronym to Unmanned Aerial Vehicle, or commonly known as drone can be remotely navigated as it has no pilot on-board. The US Military Unit introduced this aircraft during World War I in 1917 [1]. UAVs are distinguished to be vitally different from manned aircraft and cruise missiles, although they share some common features [2]. Various kinds of autonomy can be applied to the flights of UAVs such as fully autonomous programmed in on-board system, or remotely controlled by an operator on the ground [3], [4], [5]. Multirotor UAV can accomplish low-velocity flight and vertical take-off and landing compared to fixed wing aircraft. The propellers of multirotor located respectively in the front and back parts, or left and right sides and rotate in opposite directions. Multirotor UAV flight has the advantage of flexibility, good security and has a simple structure [6].

In the United States, the domestic use of UAVs is however predicted to continually push toward smaller platforms that are more affordable and more manageable. The reduced cost of UAVs has become a substantial selling point in the market and becomes more affordable for everyone to have. For example, a UAV system with a ground operating computer can cost less than USD50,000, compared to a police helicopter can cost up to USD1 million with the same functions. The continuing process of miniaturization of sensors, data links solutions, controls, and computing elements contributes to the selling point [7]. Vehicle teleoperation, means operating a vehicle at a distance, broadens the human capability of manipulating an object at a distance by accommodating the operator similar conditions to those of a remote location and to reach difficult environment, reduce cost, and avoid risking human's life [8], [9], [10]. Communication between machine and operator needs to take place under optimum conditions and information provided must be in good quality to achieve a functional teleoperation system. In this paper, a comparison has been made between different UAV control systems to find out which approach provides more efficient and effective human-robot interaction for smooth UAV navigation. Rapid response to changing sensor data is critical, as UAV are real-time systems. Drone as shown in Fig. 1 was used in the experiment.

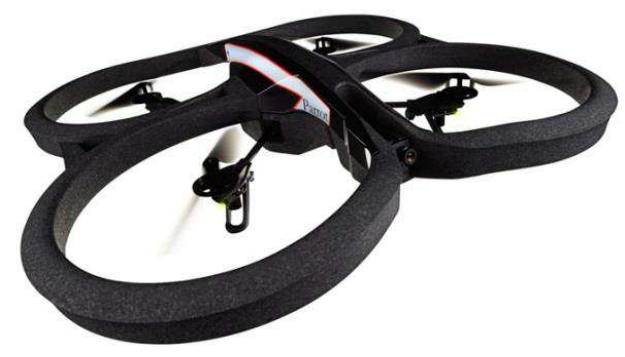

Fig. 1. Parrot AR. Drone 2.0 


\section{MATERIAL AND METHOD}

The quadrotor showed a huge advantage in disaster relief activities due to its flexibility and manoeuvrability recently. Nevertheless, the scene of in-door environment is usually complex and difficult to be detected. Since the development of ENIAC (Electronic Numerical Integrator and Computer), the first full electronic computer in 1946, computer has been used widely in science and engineering [47]. A reliable motion control and real-time pose estimation are two critical tasks for a quadrotor UAV despite its flexibility and manoeuvrability [11], [6]. Radio frequency communication in a spectrum $2.4 \mathrm{GHz}$ is required for a reliable communication system with the UAV as a pre-programmed UAV path is less flexible to handle unforeseen situation in real-time [12]. RF links hardware are inexpensive but they come with some limitations; limited range, time consuming in design and development, operation reliant on base PC, and require custom written communication protocol [48]. The electronic system control can be installed in three entities; on the UAV body, on the ground, and at the human pilot (remote control) for emergency purposes.

\section{A. UAV On-board System}

The on-board electronic system was designed to expanse the components across the UAV body in a way the gravity centre does not reallocate too much than the original platform. Installation of all electronic system in a large box under the UAV body may prevent aerobatic manoeuvres. A reliable communication is a necessary as the system components are spread apart. The high-current and highvoltage switching in UAV movements cause additional electromagnetic interferences and the communication between the modules of the control system can be disrupted. There are several options for the architecture of an on-board computer system; the ETXexpress module, the real-time module, the Inertial Measurement Unit, the System Monitor Module, the servomotors, the radio transceivers, and the vision system [13].

\section{B. Ground Based Station}

The main objective of the base station is to obtain and provide information or references to the operator and the UAV. It is an optional component of the control system as the on-board computer of the UAV can execute the control algorithm by itself. Ollero et al. [14] designed and implemented a control system for the coordination of multiple heterogeneous UAV with different autonomy degree. Shatat and Tutunji [15] discussed a practical experience in modifying an existing RC quadrotor to accept new operations. The real-time communications between the airborne and the ground computer systems will be provided by using a couple of full duplex transceivers. Cai et al. [16] tested the latest navigation technology in a nonlinear way using a radio-controlled helicopter model as a basic aircraft, a simple avionic system to implement, and a support system on the ground as shown in Fig. 2.

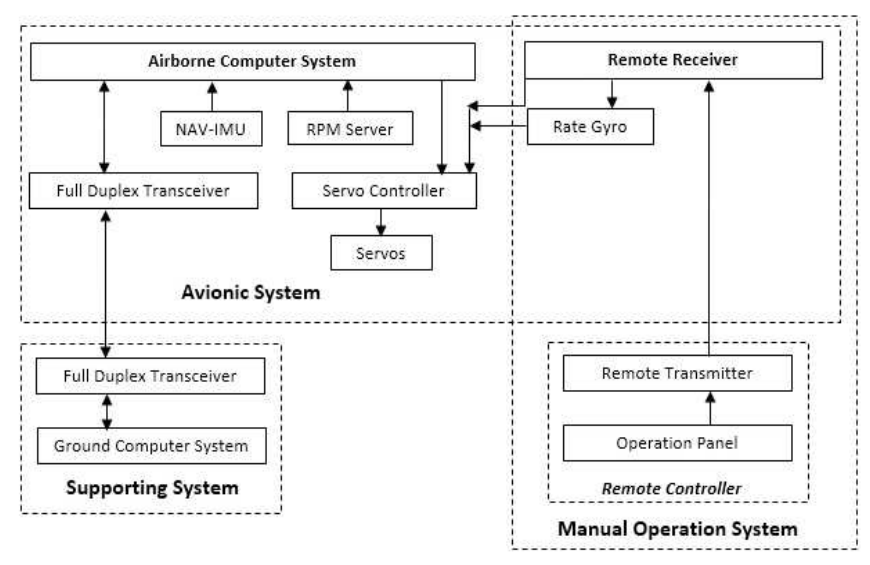

Fig. 2. Simple avionic system

\section{Human Pilot (Remote Control)}

The main objective of the base station is to obtain and provide information or references to the operator and the UAV. It is an optional component of the control system as the on-board computer of the UAV can execute the control algorithm by itself. The two major types of empowering technologies for HCI are contact- and vision-based devices. However, the user needs to be accustomed in using these devices or else these devices are difficult to handle for users with low computer literacy.

\section{1) Hand-controller:}

Human pilot involved in UAV experiments such as testing for the basic functionality platform, to trigger the automatic control functions or to take over in case of emergency. The control system needs to receive and deliver correct results in hard real-time or it would be considered as a system failure. In HRI-based system, it is a necessary for humans to be able to communicate and control robots in a natural and most efficient way especially in safe critical applications such as the military and search-and-rescue [17]. Therefore, teleoperation control fulfilled the idea. Devices that can be used to control the remote system such as radiocontroller, mouse, keyboard, joystick, haptic device, depthcamera, and touch screen. Wireless communication systems send and receive the information between control system and the UAV to be used by the pilot and the control centre [14].

Stingu and Lewis [13] discussed that UAV navigation using radio-controller as shown in Fig. 3 may contribute to irrelevant risk to the system as it is sensitive to radio response. The radio communication involves two distinct links for transmitting and receiving. The first link is transceivers with $900 \mathrm{MHz}$ band to cover about 14 mi radius. The UAV can only transmit/receive the most important flight variables to/from the ground station or remote control as it only has the maximum bitrate of $115.2 \mathrm{kbps}$. The second link is a wireless network with a high-speed of $802.11 \mathrm{n}$ and a maximum outturn at $300 \mathrm{Mbps}$. Each device may communicate with any other by the network using UDP packets. 


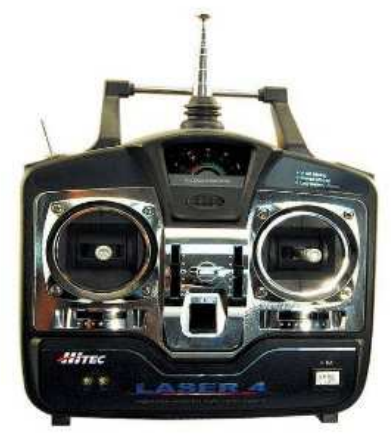

Fig. 3. Radio-controller

Computer keyboard such in Fig. 4 is seen as an alternative device to navigate UAVs. The limitations of human cognitive skills, decision-making, judgment, and tactical understanding in the use of Unmanned Aerial Vehicle cause a need to redesign the current HCI to improve the interaction and communication links between UAVs and the operator [18]. With the use of computer keyboard in addition of a screen layout, the system may test the cognitive workload of the operator and it may display information to increase situational awareness as the operator will see everything, interpret it, make relevant decision, and implement the decision.
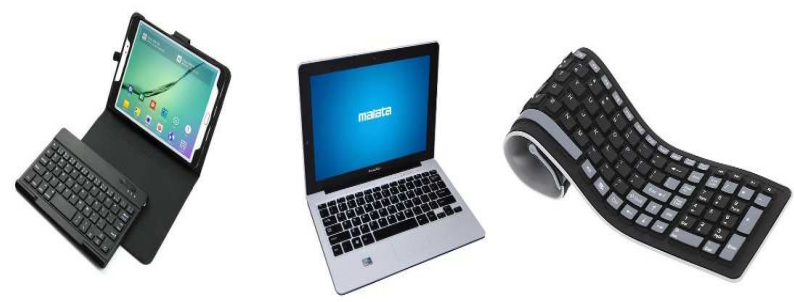

Fig. 4. Computer keyboards

There are some advantages by using haptic feedback in teleoperation; improves operator performance, the task of remote navigation in difficult environments is easier, improves the quality of motion control, and the feedback is useful for the user [9], [19], [20], [21]. Wire or wireless mouse controller receives user's instruction such as drag, click or border selection in a 2-dimentional planar interface. For touch screen on the mobile, the screen is touched using finger to execute the application functions [22].

\section{2) Gestures and Body Postures Techniques:}

Gestures and body postures techniques have been introduced to overcome the limitations of electromechanical devices. The communication based on gestures in humanrobot systems can provide an expressive, natural and intuitive approach. Gestures are intended to express messages in non-verbal or non-vocal communication of human behaviour and can be represented by a single word, a phrase, a single command, or a sequence of commands and may be dynamic or static [22], [23], [24], [25], [26], [27], [28], [29], [30]. Gestures recognition devices are including data gloves, marker, Leap Motion, and Microsoft Kinect. Data gloves are used to capture the movements of hand and fingers but they are cumbersome, complicated, expensive, have difficulties to fit in size, long-term reliability, tethered hands and it would be more complex if coloured gloves are used [31], [6], [32]. A controlled environment to obtain high quality data and excessive time for placement are the limitations for marker based method. Therefore, markerless motion approach has been introduced but could only be used when accuracy is not a necessary and motion validation and gesture movements do not have to be precise [33], [34], [35]. A controller relatively in the size of a box of matches introduced a new gesture and position tracking system, allows for the precise and fluid tracking of multiple hands, fingers, and small objects in free space with sub-milimeter accuracy, named the Leap Motion [36], [37]. The Leap Motion controller was employed as an interface for hand motion tracking without the demand of using any external instruments [49]. However, the controller has limited recognition area, sensitive, and lack of accuracy. Kinect is proficient to contribute a more attractive interaction between users and robots but there are three critical challenges in its performance; USB bandwidth, infrared noise and interference, and 3D calibration and registration [38]. Kinect which usually connected to a computer via USB provides a natural controller environment, follows the user's body movement and translated into commands to be sent to the quadrotor via wireless fidelity (wifi) [39], [40], [17].

\section{3) Vision-Based Techniques:}

Vision-based methods have been discussed in the context of autonomous landing management based on an on-board visual navigation system using inertial sensors combined with a single camera, and off-the-shelf computer hardware [41], [42]. HMDs offer ecologically relevant proprioceptive prompts to operator [43, 46]. In a helicopter or fighter aircraft, a HMD not only deliver the relevant positional and situational information to the pilot in his line of sight, but it also allows him to control sensors and weapons with his head movements [44]. Vision-based approach is based on how humans obtain information about their surroundings. Nonetheless, this approach may be the most difficult to implement. The main constraint in vision-based gesture recognition is the large variety of existing gestures. Gestures under different lighting conditions and cluttered surroundings may affect the recognition robustness [45].

An experiment was executed to measure the effects of using a Helmet Mounted Display versus a conventional computer monitor and joystick to control UAV, and the subjective measure including accuracy and responses, workload, fatigue, simulator sickness, and situational awareness [43].

\section{RESULT AND DISCUSSION}

Experiments on human-piloted or remotely-controlled techniques of UAV navigation have been executed for performance measure. Parrot AR.Drone came with complete basic hardware including battery, camera, and wearable frame. All of those were needed in the experiment. The wearable frame was an optional but it is recommended to place it in the drone to avoid any physical contacts or accidents with any hard objects around. The camera has the ability to capture images or record videos in real-time and 
store it in the blackbox or display it on the screen while the drone is in flying mode. The battery can last around eight to eleven minutes. A task completion by a person could take two to five minutes thus a spare battery may well needed. First experiment involved a human operator navigating the UAV using computer keyboard. Keys on computer keyboard work as the navigator to fly the UAV. Android AR.FreeFlight application was used to replace radiocontroller in this experiment. The application can be downloaded and installed in a smartphone. A computer or a laptop could be heavier than a radio-controller or an android smartphone, but it could provide more functions and benefits in emergency when debugging is required. The operator can determine and analyse the problem and solve it within the time. The experiments were executed outdoor to provide more flexible and extensive environment to measure the UAVs performance. The tasks were to take-off, hover, fly downward, upward, forward, backward, to the left, to the right, and land the UAV. The user worked on the computer while it connected to the UAV via wi-fi as shown in Fig. 5.

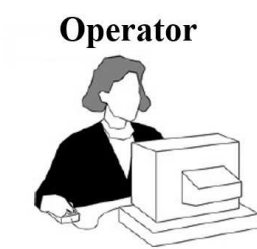

Control Station

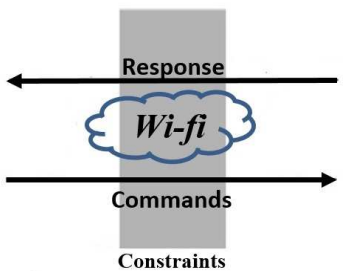

(Surroundings, distance, etc.)

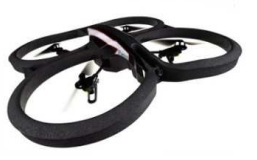

UAV
Fig. 5. Application structure

Arrow keys were set to give the functions of fly forward, backward, to the left and to the right. 'Page Up' key to fly upward, 'Page Down' key to fly downward, 'T' key for take-off and 'L' key for landing the UAV. Fig. 6 shows the common keys used to ease the navigation and provide better understanding of the functions. An interface layout was designed to accommodate the user to see the view from the UAV's camera but the interface was not the critical issue in the performance measure.

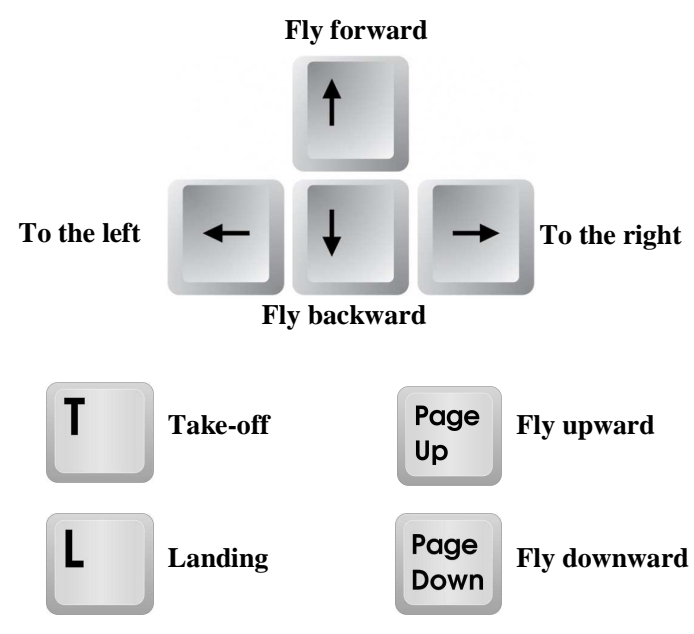

Fig. 6. UAV navigation keys on computer keyboard
Fig. 7 until Fig. 10 show the testing and validation process. Fig. 7 shows the UAV can fly up to four metre high and an outdoor environment provide more flexible surrounding for the experiments compared to indoor. Weather is one of the factors to ensure the experiment to run smoothly. Rainy or too much wind would affect the UAV to fly accordingly. The users need to test and validate all the keys and functions on the computer keyboard to compare with the UAV manoeuvrability using RC.

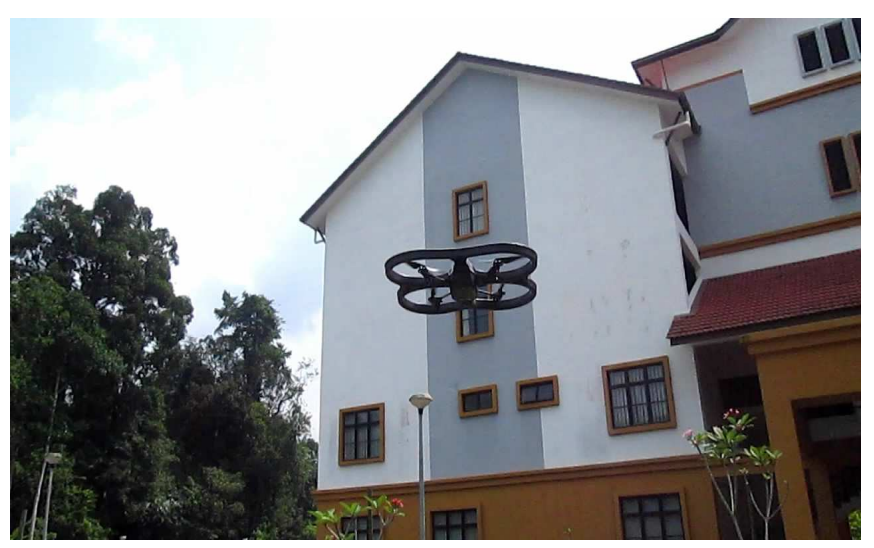

Fig. 7. The UAV flies up to four metre high

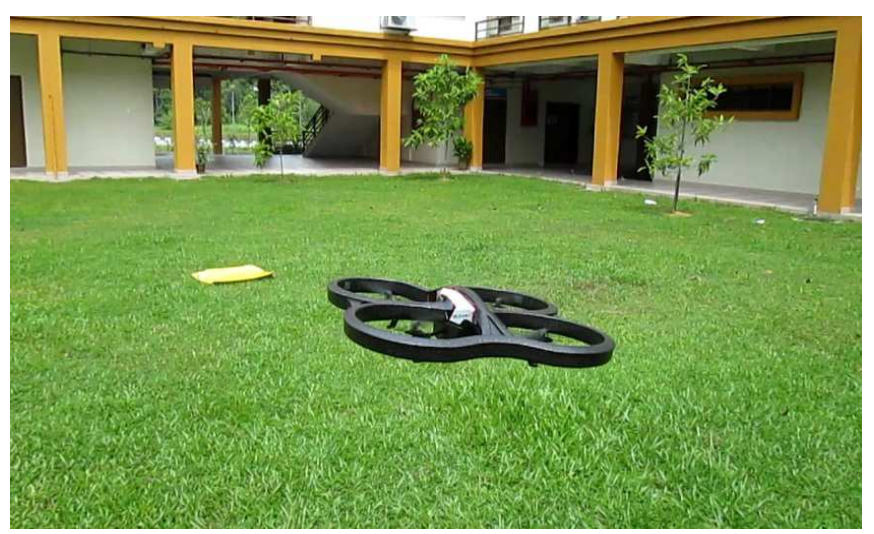

Fig. 8. Stability to hover

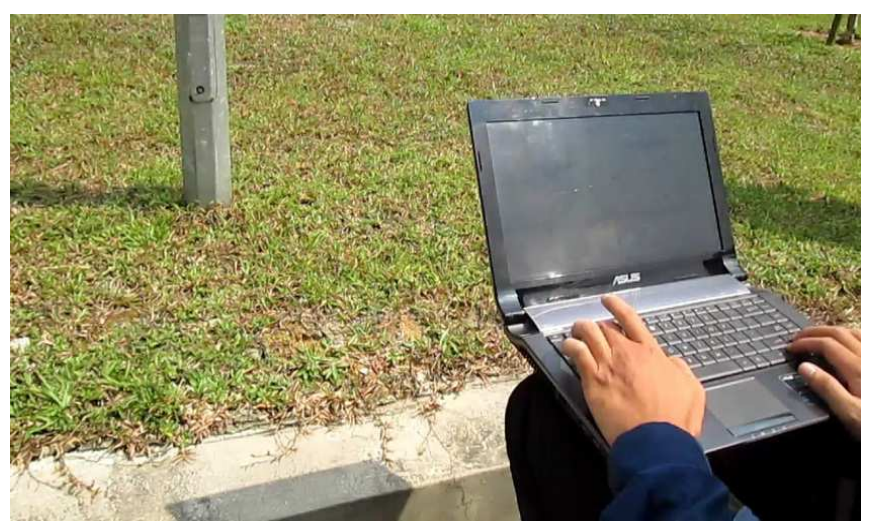

Fig. 9. Efficiency in controlling 


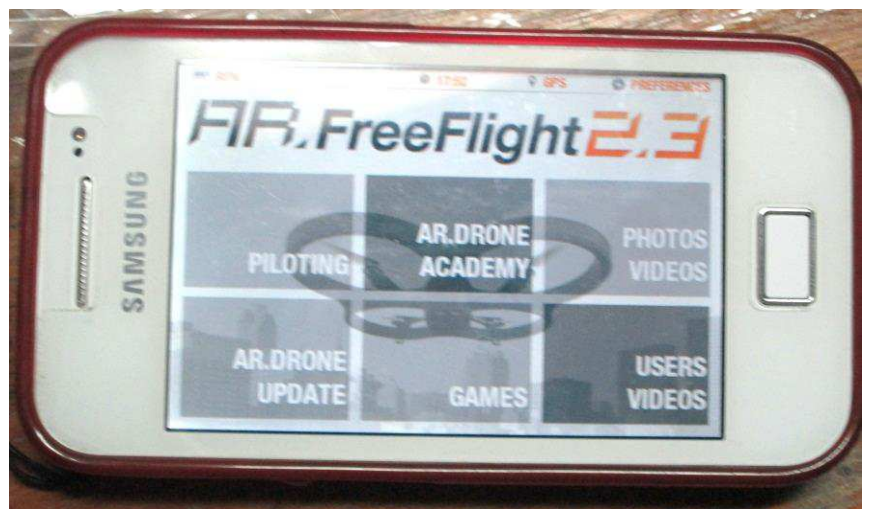

Fig. 10. Android AR.FreeFlight 2.3 application

Table 1 summarized the comparison between radiocontroller and computer keyboard in UAV navigation experimented by five IT students and two UAV researchers. Scale 1 to 5 indicates very poor to very good. The record consisted of time, stability, manoeuvrability, accuracy, and user friendliness. The experiments were conducted outdoor to provide wide-space environment for better response. It shows that computer keyboard has better performance than radio-controller in navigating $\mathrm{UAVs}$ due to $\mathrm{RC}$ is frequencysensitive and likely got more interference. Moreover, an added interface in the system conducted by computer keyboard may produce more flexible environment to the user.

TABLE I

RADIO-CONTROLLER VS. COMPUTER KEYBOARD

\begin{tabular}{|c|c|c|c|c|c|c|c|c|c|c|}
\hline & \multicolumn{3}{|c|}{ Radio-controller } & \multicolumn{5}{c|}{ Computer keyboard } \\
\hline & 1 & 2 & 3 & 4 & 5 & 1 & 2 & 3 & 4 & 5 \\
\hline $\begin{array}{c}\text { Time/Speed } \\
\text { (task completion) }\end{array}$ & & & $\checkmark$ & & & & & & $\checkmark$ & \\
\hline Stability & & $\checkmark$ & & & & & & & $\checkmark$ & \\
\hline Maneuverability & & & & $\checkmark$ & & & & & $\checkmark$ & \\
\hline Accuracy & & & $\checkmark$ & & & & & & $\checkmark$ & \\
\hline User friendliness & & $\checkmark$ & & & & & & $\checkmark$ & & \\
\hline Layout & & $\checkmark$ & & & & & & $\checkmark$ & & \\
\hline
\end{tabular}

Sanna et al. [17] performed an evaluation using three different input devices to navigate UAV; joystick, iPhone, and Kinect. An experiment using three different approaches; traditional, haptic, and gesture, produced a significant result to one another. A group of users needed to complete flight session from take-off to landing in an area of $56 \mathrm{~cm} \times 56 \mathrm{~cm}$ in size. Marker for pose estimation was used to set the perimeter for the UAV flight area. Uncompleted, semicompleted and completed indicate the success of users to accomplish the tasks. Table 2 shows $100 \%$ task completion using Kinect, $50 \%$ using joystick, and $25 \%$ using iPhone. Average time recorded for Kinect is 61.5 seconds, which was the longest time for task completion, but it achieved the highest percentage amongst other two devices.
TABLE II

EXPERIMENTAL RESULTS USING THREE DEVICES

\begin{tabular}{|l|c|c|c|c|}
\hline \multicolumn{1}{|c|}{ Device } & $\begin{array}{c}\text { Average } \\
\text { time (s) }\end{array}$ & $\begin{array}{c}\text { Un } \\
\text { completed } \\
\text { \% }\end{array}$ & $\begin{array}{c}\text { Semi- } \\
\text { completed \% }\end{array}$ & $\begin{array}{c}\text { Completed } \\
\%\end{array}$ \\
\hline iPhone & 45.25 & 62.5 & 12.5 & 25 \\
\hline Joystick & 27 & 12.5 & 37.5 & 50 \\
\hline Kinect & 61.5 & 0 & 0 & 100 \\
\hline
\end{tabular}

Hasan and Abdul-Kareem [45] experimented gesture recognition with contact- and vision-based approaches for better HCI performance. The exploitation of gestures to deliver information is an important part of human communication. Contact-based devices involving physical interaction of users with the interfacing device. The users should be accustomed to this approach as it provide more natural way in conducting a system, nonetheless, it could be difficult for users with low computer literacy and bad health condition in simulator experience especially feeling nausea.

The comparison between contact- approach and visionapproach is shown in Table 3. User-independence produces an environment where different users can control the system and recognize human gestures in different colours and sizes. Contact-based devices have high recognition accuracy and less complex implementation but may be uncomfortable to the users. On the other hand, vision-based devices have configuration complexity and occlusion but more userfriendly. However, vision-based approach is probably the most difficult to implement.

TABLE III

COMPARISON BETWEEN CONTACT- AND VISION-BASED APPROACHES

\begin{tabular}{|l|c|c|}
\hline \multicolumn{1}{|c|}{ Criterion } & Contact- approach & Vision- approach \\
\hline User cooperation & Yes & No \\
\hline User intrusive & Yes & No \\
\hline Precise & Yes/No & No/Yes \\
\hline Flexible to configure & Yes & No \\
\hline Flexible to use & No & Yes \\
\hline Occlusion problem & No (Yes) & Yes \\
\hline Health issues & Yes (No) & No \\
\hline
\end{tabular}

Morphew et al. [43] analysed the comparison results between HMD and computer monitor using ANOVAs and non-directional (two-tailed) T-test. A graph has been developed as shown in Fig. 11 to Fig. 13 to summarize the analysis. Three critical measurements were recorded in the graphs; target detection accuracy, cursor distance angle, and effect measurement of HMD and computer monitor usage. Eight male undergraduates voluntarily participated in the experiment and were pre-screened for both physical and visual pre-disposers to physical injury and simulator sickness. The pre-screened consisted in interviews and questionnaires regarding subjects' health history. If a subject failed to meet the minimum criteria, they were eliminated from the subject pool. The experiment was a repeated 
measures and the main objective performance measure was the user's target detection accuracy. It was calculated based on percentage of hits, misses, false alarms, and correct rejections. Measurement for tracking accuracy involved cursor distance and slant range. The HMD and CRT displays resulted in comparable target classification accuracy, but findings in the HMD conditions discovered subsided operator targeting accuracy and boosted simulator sickness; nausea, eye strain, and disorientation. The eyestrain experienced by the subjects using HMD is a common effect due to exposure to virtual environments.

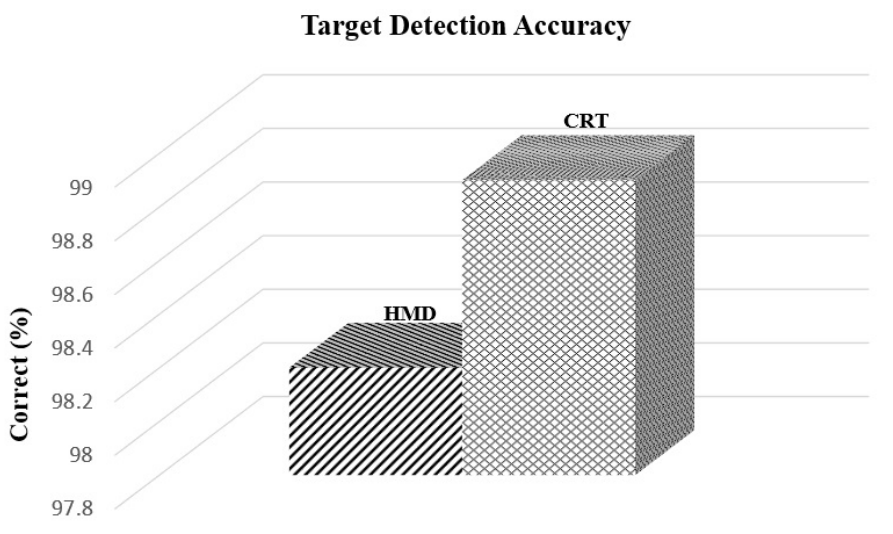

Fig. 11. Target detection accuracy by display [17]

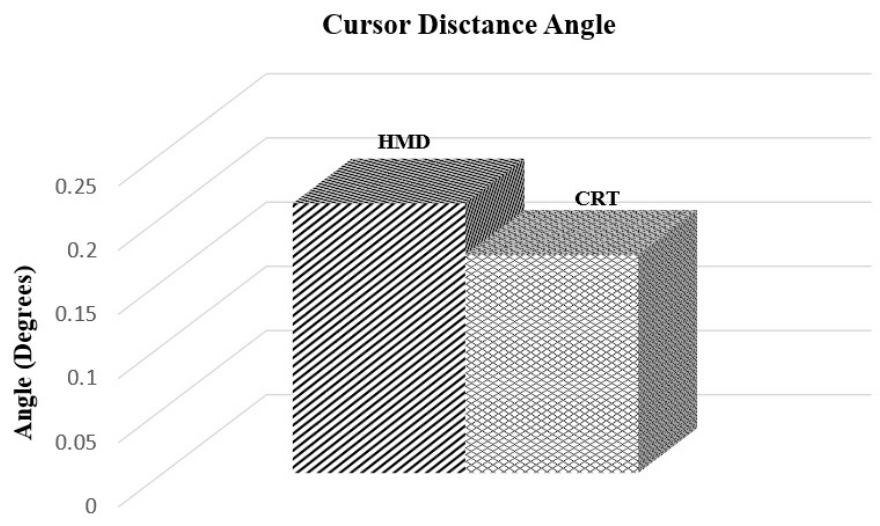

Fig. 12. Cursor distance angle by display [17]

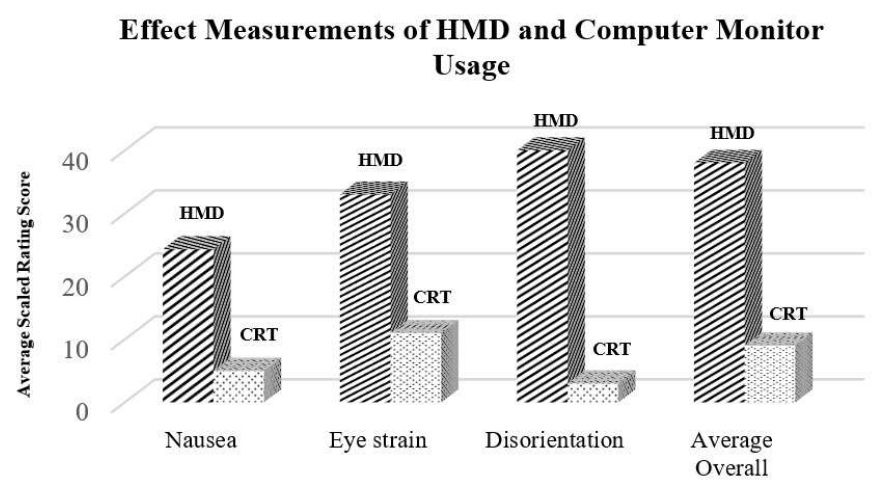

Fig. 13. Effect measurement of HMD and computer monitor usage [17]

\section{CONCLUSIONS}

Recently, the development of multirotor UAV is increasing immensely. The research and usage of UAV in many applications in various fields proved the statement. Modelling and stabilization system especially in UAV navigation are still the obvious challenges in the field. There were many accidents reported related to UAV either it is controlled by autonomous programmed or remotely navigated by the operator. The data and information from the experiments need to be collected for analysis; skill development time, error tracking, and user satisfaction level ratings. The main purpose of this research is to review related works in Unmanned Aerial Vehicle navigation especially in human-piloted devices to provide better HCI performance. Human-machine interaction should be efficient and effective for better performance and better understanding between user and machine. Human-piloted or remote-controlled approach in UAV navigation has been highlighted in this paper as on-board system is usually installed in default at every UAV purchase and ground control system is only optional. Best methods or device can only be chosen based on the situation and environment. For common and cheaper device, computer keyboard with an addition of system interface provides good performance. It is more accurate as the operator works only with buttons on keyboard where the flying coordinate and data reading is less sensitive than using radio-controller. It could be used for surveillance and more organised task. Kinect is best used for gesture approach as it can recognize the whole body for six players and up to 20 joints per player. Users with low computer literacy would be more comfortable in using gesture approach, as it is more user friendly and flexible. HMD can provide for AR and VR experience to the user but it comes with several challenges especially in health condition. This research is open for further discussion in future works.

\section{ACKNOWLEDGMENT}

This research is supported by Ministry of Higher Education Malaysia Research Grant Scheme of FRGS/2/2013/ICT01/UKM/02/4.

\section{REFERENCES}

[1] Valavanis, K.P.: Advances in Unmanned Aerial Vehicles : State of the Art and the Road to Autonomy (2007)

[2] Weidlich, C., Altmann, J., Anastasakis, I., Pedatzur, R., Shulga, O., Stroh, P., Wezeman, P.: Unamnned Aerial Vehicle; A Challenge to a WMD/DVs Free Zone in the Middle East (2012)

[3] Hansen, J.P., Mollenbach, E., Alapetite, A., MacKenzie, I.S., Mulvey, F.B.: The Dawn of Personal Drone. In: CHI'13, Paris, France (2013)

[4] Prabuwono, A.S., Triharminto, H.H.: Multirotor Unmanned Aerial Vehicle: A Review. In: 3rd Makassar International Conference on Electrical Engineering and Informatics, pp. 19-23, Indonesia (2012)

[5] Mortezapoor, S., Taale, M., Soleimani, S.: A Multi Modal Interface Approach to Control an Unmanned Aerial Vehicle. In: Swiss Joint Master of Science in Computer Science - Summer Semester, Bern, Switzerland (2012)

[6] Zhang, Y., Mu, X., Wang, Y.: Pose Control of Quadrotor Unmanned Aerial Vehicle Based on Double Filters. In: IEEE International Conference on Cyber Technology in Automation, Control and Intelligent Systems, pp. 298-304, Shenyang (2015)

[7] Cavoukian, A.: Privacy and Drones: Unmanned Aerial Vehicle. Canada (2012) 
[8] Draper, M.H., Ruff, H.A.: Multi-Sensory Displays and Visualization Techniques Supporting the Control of Unmanned Air Vehicles (2000)

[9] Boboc, R.G., Moga, H., Talaba, D.: A Review of Current Applications in Teleoperation of Mobile Robots. In: Bulletin of the Transilvania Uni-versity of Brasov, Series I: Engineering Sciences, Vol. 5 (54) No. 2 (2012)

[10] Fong, T., Thorpe, C.: Vehicle Teleoperation Interfaces. In: Autonomous Robots 11, Kluwer Academic Publishers, pp. 9-18 (2001)

[11] Lin, X., Zhuang, Y., Zhao, Y.: Real-time Pose Estimation and Motion Control for a Quadrotor UAV. In: 11th World Congress on Intelligent Control and Automation, pp. 2370-2375, Shenyang (2014)

[12] Schwaerzler, H. J.: U.S. Patent No. 6,377,875. Washington, DC: U.S. Patent and Trademark Office (2002)

[13] Stingu, E., Lewis, F.L.: A Hardware Platform for Research in Helicopter UAV Control. In: Springer - J Intell Robot Syst, pp. 387406 (2009)

[14] Ollero, A., Alcazar, J., Cuesta, F., Lopex-Pichaho., F., Nogales, C.: Helicopter Teleoperation for Aerial Monitoring in the COMETS Multi-UAV System. In: European Commission in the IST Programme, Coimbra, Portugal (2003)

[15] Shatat, A., Tutunji, T.A.: UAV Quadrotor Implementation: A Case Study. In: 11th International Multi-Conference on Systems, Signals and Devices, pp. 1-6, Barcelona (2014)

[16] Cai, G., Peng, K., Chen, B.M., Lee, T.H.: Design and Assembling of a UAV Helicopter System. In: 2005 International Conference on Control and Automation, pp. 697-702, Budapest, Hungary (2005)

[17] Sanna, A., Lamberti, F., Paravati, G., manure, F.: A Kinect-based Natural Interface for Quadrotor Control. In: Entertainment Computing, http://dx.doi.org/10.1016/j.entcom.2013.01.001 (2013)

[18] Matsangas, P., McCauley, M.E.: Small Unmanned Aerial Vehicles: The Way Forward. In: Network Centric Warfare Conference, Athens, Greece (2005)

[19] Lee, S., Sukhatme, G., Kim, G.J., Park, C.M.: Haptic Teleoperation of a Mobile Robot: A User Study. In: Presence: Teleoperators and Virtual Environments 14, No. 3, pp. 345-365 (2005)

[20] Farkhatdinov, I., Ryu, J.H., An, J.: A Preliminary Experimental Study on Haptic Teleoperation of Mobile Robot with Variable Force Feedback Gain. In: Proceedings of IEEE Haptics Symposiums, pp. 251-256 (2010)

[21] Nadrag, P., Temzi, L., Arioui, H., Hoppenot. P.: Remote Control of an Assistive Robot Using Force Feedback. In: Advanced Robotics (ICAR), 2011 15th International Conference on IEEE, pp. 211-216 (2011)

[22] Wu, H.H., Bainbridge-Smith, A.: Advantages of Using a Kinect Camera in Various Applications (2011)

[23] Koceski, S., Koceska, N.: Vision-based Gesture Recognition for Human-Computer Interaction and Mobile Robot's Freight Ramp Control. In: Proceedings of the ITI 2010 32nd Int. Conf. on Information Technology Interfaces, June 21-24. Cavtat, Croatia. pp. 289-294 (2010)

[24] Murthy, G.R.S., Jadon, R.S.: Hand Gesture Recognition Using Neural Networks. In: IEEE 2nd International Advance Computing Conference. pp. 134-138 (2010)

[25] Ye, G., Corso, J.J., Hager, G.D.: Gesture Recognition Using 3D Appearance and Motion Features. In: Proceedings of the 2004 IEEE Computer Society Conference on Computer Vision and Pattern Recognition Worshops (CVPRW'04). pp. 160-167 (2004)

[26] Cohen, I., Li, Hongxia.: Inference of Human Postures by Classification of 3D Human Body Shape. In: International Workshop on Analysis and Modeling of Faces and Gestures. pp. 74-81 (2003)

[27] Gavrila, D.M. and Davis, L.S. 1995. Towards 3-D Model-Based Tracking and Recognition of Human Movement: A Multi-View Approach. International Workshop on Automatic Face- and GestureRecognition. pp.272-277 (1995)

[28] Mo, Hao-Cheng, Leou, Jin-Jang, Lin, Cheng-Shian.: Human Behavior Analysis Using Multiple 2D Features and Multicategory Support Vector Machine. In: Proceedings of the International MVA2009 IAPR Conference on Machine Vision Applications, May 20-22. pp. 46-49 (2009)

[29] Patsadu, O., Nukoolkit, C., Watanapa, B.: Human Gesture Recognition Using Kinect Camera. In: Computer Science and Software Engineering (JCSSE), 2012 International Joint Conference. pp. 28-32 (2012)
[30] Jais, H.M., Mahayuddin, Z.R., Arshad, H.: A Review on Gesture Recognition Using Kinect. In: IEEE International Conference on Electrical Engineering and Informatics, pp. 594-599, Indonesia (2015)

[31] Wachs, J.P., Stern, H., Edan, Y.: Optimization of Hand Gesture Command Vocabularies - A Multiobjective Quadratic Assignment Approach (2004)

[32] Kulkarni, V.S., Lokhande, S.D.: Appearance Based Recognition of American Sign Language Using Gesture Segmentation. In: International Journal on Computer Science and Engineering (IJCSE). vol. 2. no. 3. pp. 560-565 (2010)

[33] Baena, A.F., Susin, A., Lligadas, X.: Biomechanical Validation of Upper-body and Lower-body Joint Movements of Kinect Motion Capture Data. In: Intelligent Networking and Collaborative Systems (INCoS), 2012 4th International Conference. pp. 656-661 (2012)

[34] Choppin, S., Wheat, J.: Marker-less Tracking of Human Movemen Using Microsoft Kinect. In: 30th Annual Conference of Biomechanics in Sports. pp. 231-234 (2012)

[35] Kulshreshth, A., Zorn, C., Laviola Jr., J.J.: Poster: Real-time Markerless Kinect Based Finger Tracking and Hand Gesture Recognition for HCI. In: IEEE Symposium on 3D User Interfaces 2013. pp. 187-188 (2013)

[36] Weichert, F., Bachmann, D., Rudak, B., Fisseler, D.: Analysis of the Accuracy and Robustness of the Leap Motion Controller. In: Sensors, 13(5), pp. 6380-6393 (2013)

[37] Guna, J., Jakus, G., Pogačnik, M., Tomažič, S., Sodnik, J.: An Analysis of the Precision and Reliability of the Leap Motion Sensor and Its Suitability for Static and Dynamic Tracking. In: Sensors, 14(2), pp. 3702-3720 (2014)

[38] Hossny, M., Fillipidis, D., Abdelrahman, W., Zhou, H., Fielding, M. Mullins, J., Wei, L., Creighton, D., Puri, V., Nahavandi, S.: Low Cost Multimodal Facial Recognition via Kinect Sensors. In: Land Warfare Conference 2012: Potent Land Force for a Joint Maritime Strategy, pp. 77-86 (2012)

[39] Boudjit, K., Larbes, C., Alouache, M.: Control of Flight Operation of a Quad rotor AR. Drone Using Depth Map From Microsoft Kinect Sensor. In: International Journal of Engineering and Innovative Technology, 3(3), 15-19 (2013)

[40] Asiimwe, R., Anvar, A.: Automation for the Maritime UAV Command, Control, Navigation Operations, Simulated in Real-Time Using Kinect Sensor: A Feasibility Study. In: World Academy of Science, Engineering and Technology. pp. 22-26 (2012)

[41] Merz, T., Duranti, S., Conte, G.: Autonomous Landing of an Unmanned Helicopter Based on Vision and Inertial Sensing. In: Experimental Robotics IX, Springer Tracts in Advanced Robotics, vol. 21, pp. 343-352 (2006)

[42] Cesetti, A., Frontoni, E., Mancini, A., Zingaretti, P., Longhi, S.: A Vision-Based Guidance System for UAV Navigation and Safe Landing Using Natural Landmarks. In: Selected papers from the 2nd International Symposium on UAVs, Reno, Nevada, USA June 8-10, Springer Netherlands, pp. 233-257 (2009)

[43] Morphew, M. E., Shively, J. R., Casey, D.: Helmet-Mounted Displays For Unmanned Aerial Vehicle Control. In: Defense and Security, International Society for Optics and Photonics, pp. 93-103 (2004)

[44] Brandtberg, H., Segerhammar, P., Waldewof, C.: Head-Mounted Display. In: Ericsson Review No. 1, pp. $41-49$ (1997)

[45] Hasan, H., Abdul-Kareem, S.: Human-computer Interaction Using Vision Based Hand Gesture Recognition Systems: A Survey. In: Neural Computing \& Application (2013)

[46] Draper, M. H., Ruff, H. A., Fontejon, J. V., Napier, S.: The Effects of Head-Coupled Control and a Head-Mounted Display (HMD) on Large-Area Search Tasks. In: Proceedings of the Human Factors and Ergonomics Society Annual Meeting, SAGE Publications, Vol. 46, No. 26, pp. 2139-2143 (2002)

[47] Clifton, J.R., Fronhsdorff, G.: Applications of Computers and Information Technolo-gy. In: Analytical Techniques in Concrete Science and Technology: Principles, Tech-niques and Ap-plications, pp. 765-799, Gaithersburg, USA (2001)

[48] Poh Eng Fong, Mohd Amaluddin Yusoff: Real-Time Control of WiFi Surveillance Robot. In: International Journal on Advanced Science, Engineering and Information Technology, vol. 1, no. 4, pp. 423-426 (2011)

[49] Fazlur Rahman Khan, Huey Fang Ong, Nurhidayah Bahar.: A Sign Language to Text Converter Using Leap Motion. In: International Journal on Advanced Science, Engineering and Information Technology, vol. 6, no. 6 (2016) 